\title{
Letter processing during eye fixations in visual search
}

\author{
KEITH RAYNER and DONALD L. FISHER \\ University of Massachusetts, Amherst, Massachusetts
}

\begin{abstract}
In three experiments, subjects searched from left to right for a target letter in a horizontal array as a window moved in synchrony with their eyes. Wherever the subject looked within the window, letters from the search array were available for processing; outside the window, each letter in the array was replaced by an X (in Experiments 1 and 2) or by some different letter (in Experiment 3). In all three experiments, subjects were held to a high level of accuracy. Experiment 1 indicated that there are two regions of processing, one central, or decision, region and a second more eccentric, or preview, region. Experiment 2 indicated that the sizes of both the decision and preview regions increase as the target is made more dissimilar to the distractors. Furthermore, the results of the experiment suggested that more than just location information (information about where next to move the eyes) is obtained from the preview region. Finally, Experiment 3 indicated that, although the additional information subjects gain from the preview region includes partial letter information, this information cannot be used to make a decision about the absence of a target. In short, there appear to be two qualitatively different regions in visual search: a decision region, where information about the presence/absence of a target is available, and a preview region, where partial letter information is available but where information on the absence of a target is not available.
\end{abstract}

As in reading, the visual search of a display typically requires that subjects make a number of eye movements. And as in reading, information in parafoveal vision may influence processing in the more central foveal region. However, although much is known about the influence of parafoveal information in reading (Rayner, 1984), very little is known about the influence of parafoveal information in visual search tasks in which subjects search for a target letter.

The primary goals of this research were twofold, one theoretical and one methodological. For theoretical purposes, an attempt was made to determine whether, during search for a target letter, the visual field could be partitioned into two identifiably different regions both of which influence processing, one a central region and one a more eccentric region. The central, or decision, region includes each letter that subjects can analyze in enough detail to decide whether it is or is not a target. The eccentric, or preview, region includes all stimuli outside of the decision region that, in some measurable way, influence processing within the decision region. According to such a distinction, as the eye fixates on a given region, low-level visual feature analyses (Brand, 1971; Ingling, 1972; Neisser \& Beller, 1965) might be carried out for letters appearing parafoveally, in the preview region, while more complex analyses are carried out on

This research was supported by Grant MH 39960 from the National Institute of Mental Health. We would like to thank Charles Clifton, Susan Duffy, Charles Eriksen, Kevin O'Regan, Alexander Pollatsek, Arnold Well, and an anonymous reviewer for their valuable comments. Requests for reprints should be sent to Keith Rayner, Department of Psychology, University of Massachusetts, Amherst, MA 01003. letters within the decision region (where acuity is better than in the preview region). Thus, different types of information are obtained from the two regions but processing activities occur for both. We shall refer to the conjunction of the decision and preview regions as the region of effective processing.

The second, more methodological, goal was to determine whether the two putative regions could be identified using a variation of the moving window paradigm first introduced by McConkie and Rayner (1975) to study the reading process (DenBuurman, Boersema, \& Gerrisen, 1981; Ikeda \& Saida, 1978; O'Regan, 1979; Rayner \& Bertera, 1979; Rayner, Inhoff, Morrison, Slowiaczek, \& Bertera, 1981; Rayner, Well, \& Pollatsek, 1980; Rayner, Well, Pollatsek, \& Bertera, 1982; Underwood \& McConkie, 1985). In this variation, we asked subjects to search for a target letter as their eye movements were recorded. Wherever a subject looked, a window moved in synchrony with the eye movements. Around the fixation point, within the window region, all of the characters from the stimulus array were available for processing. Outside the window, the characters were replaced by other irrelevant characters. For example, in Experiment 1 , outside the window area all of the letters from the search array were replaced by Xs. Thus, we were able to control the number of letters that were available for processing on each fixation.

The above two goals were addressed in three related experiments. In Experiment 1, subjects were asked to search for a target letter in a background of distractor letters. An attempt was made to determine whether the two regions defined above (i.e., the decision and preview 
regions) were indeed distinct. In Experiment 2, the targetdistractor similarity was varied within the window only. Specifically, letters within the window were either all similar or all dissimilar to the target; the letters outside the window were all replaced by Xs. An attempt was made to determine whether the sizes of both the decision and preview regions changed in the predicted fashion. In addition, an attempt was made to determine whether information obtained from the preview region went beyond location information (i.e., information about where next to place the eyes). Finally, in Experiment 3 the similarity of the distractors to the target was varied both within and outside the window (e.g., in one condition, the distractors within the window were similar to the target, whereas the distractors outside the window were not similar to the target). An attempt was made to determine more exactly both what information in addition to location information subjects obtain in the parafoveal preview region and whether this information can be used to make a decision about the absence of a target.

\section{GENERAL METHOD}

\section{Subjects}

Six members of the University of Massachusetts community were paid to participate in the experiments. All had normal, uncorrected vision, and all had participated in previous eye-movement experiments. Prior to participating in the experiments described here, they participated in a number of practice sessions.

\section{Procedure}

When a subject arrived for an experiment, a bite bar that eliminated head movement during the experiment was prepared. Then the initial calibration of the eye-movement recording system took place. This initial calibration was generally completed in less than $5 \mathrm{~min}$. At the beginning of each session, the subject went through a set of $\mathbf{4 0}$ practice trials. The practice trials consisted of sample conditions from the particular experiment being run. Following the practice trials, the subjects were asked to search for the target letter. In all of the experiments, a consistent mapping task (Fisher, 1984; Schneider \& Shiffrin, 1977) was used in which the subjects searched for the letter $b$ or the letter $y$. On alternate days, subjects searched for $a b$ or a $y$; when the $b$ was the target, the $y$ was never used as a distractor, and vice versa. Subjects pushed a button indicating whether or not the target was present in the array; the buttonpress terminated the array. The disappearance of the stimulus array was followed by the appearance of three calibration targets (crosses). A fourth cross that moved in synchrony with the subject's eyes was also present. The experimenter asked the subject to fixate the center calibration target. If one cross was superimposed on the other, the experimenter instructed the subject to move to the leftmost calibration target. When the subject had done so, the experimenter pushed a button, which resulted in the presentation of the next stimulus array.

\section{Materials and Apparatus}

Letter strings consisting of 24 letters arranged horizontally were used as stimulus arrays. The 24 letters were divided into four string lengths of $2,4,6$, or 8 letters per string. Thus, a given stimulus array consisted of 12 2-letter strings, 6 4-letter strings, 4 6-letter strings, or 3 8-letter strings. In each block of trials (with separate blocks for each of the target letters), half of the arrays contained the target letter and half did not. When the target letter was present in the array, its location in the array was determined randomly. In addition to varying the string size, we also varied the size of a window that moved in synchrony with the subject's eyes. In all of the conditions, all of the spaces between strings were preserved. In three of the conditions, however, the number of letters available to the left and right of fixation varied. In the 15 condition, only letters from the currently fixated string were available; all other letters were replaced (by Xs in Experiments 1 and 2 and by different letters in Experiment 3). In the $2 S$ condition, all letters in the currently fixated string and all letters in the string immediately to the right of fixation were available. In the $3 \mathrm{~S}$ condition, all letters in the currently fixated string and all letters in the two strings to the right of fixation were available. Finally, in the NW (no window) control condition, the entire string was presented normally without any window constraint. Table 1 shows sample strings from Experiment 1 on two consecutive fixations. Note that when the subject made a rightward eye movement, the appropriate letters were immediately available around the new fixation point. If the subject made a leftward movement, the window also moved to the left. The number of lenters available to the right of fixation was determined by the size of the window and the string size on the current trial.

The letter strings were displayed on a Hewlett-Packard 1300A cathode ray tube (CRT), which has a P-31 phosphor with the characteristic that removing a character results in a drop to $1 \%$ of maximum brightness in $.25 \mathrm{msec}$. The letters were printed in lower case on the CRT. A black theater gel covered the CRT so that the letters appeared clear and sharp to the subjects.

Eye-movement recording was accomplished by using a Stanford Research Institute Dual Purkinje eyetracker, which has a resolution of $10 \mathrm{~min}$ of arc and a linear output over the visual angle $\left(8^{\circ}-12^{\circ}\right)$ occupied by the letter strings. The eyetracker and the CRT were interfaced to a Hewlett-Packard 2100 computer that controlled the experiment. The signal from the eyetracker was sampled every millisecond, and the position of the eye was determined every $4 \mathrm{msec}$. The display change associated with each eye movement was accomplished within $5 \mathrm{msec}$ of the completion of the saccade. The computer kept a complete record of the duration, sequence, and location of each fixation.

In the experiments, the subject's eye was $46 \mathrm{~cm}$ from the CRT and three character spaces equaled $1^{\circ}$ of visual angle. Eye move-

Table 1

Sample Stimulus Arrays from Experiments 1 and 2

\begin{tabular}{|c|c|c|c|c|c|c|}
\hline $\begin{array}{c}\text { Window } \\
\text { Size }\end{array}$ & \multicolumn{5}{|c|}{ Stimulus Arrays } & \\
\hline \multicolumn{7}{|c|}{ Experiment 1} \\
\hline NW & rth n & kpoq & hwsj & ef $v \mathrm{c}$ & opxz & mlph \\
\hline NW & rthm & kpoq & hwsj & ef $v c$ & opxz & mlph \\
\hline 1S & $\mathbf{X X X X}$ & kpoq & $\mathbf{x x x x}$ & $\mathbf{X X X X}$ & $\mathbf{x x x x}$ & $\mathbf{x x x x}$ \\
\hline is & $\mathbf{X X X X}$ & $\mathrm{xxxx}$ & hwsj & $\operatorname{xxxx}$ & $\mathbf{X X X X}$ & $\mathbf{X X X X}$ \\
\hline $2 S$ & $\mathrm{xxxx}$ & $\mathrm{kpoq}$ & hwsj & $\operatorname{XXXX}$ & $\mathbf{X X X X}$ & $\mathbf{X X X X}$ \\
\hline $2 S$ & $\mathbf{x x x x}$ & $x \dot{x} x x$ & hwsj & ef $v c$ & $\mathbf{x x x x}$ & $\mathbf{x x x X}$ \\
\hline $3 S$ & $\mathrm{XXXX}$ & kpoq & $h w \dot{s} j$ & ef $\mathrm{fuc}$ & $\mathbf{X X X X}$ & $\mathbf{x x x X}$ \\
\hline 35 & $\mathrm{XXXX}$ & $\mathbf{x} \dot{x} x \mathbf{x}$ & hwșj & ef $v \mathrm{c}$ & opxz & $\mathbf{x x x X}$ \\
\hline \multicolumn{7}{|c|}{ Experiment 2} \\
\hline \multicolumn{7}{|l|}{ Similar } \\
\hline NW & $\mathbf{p q j} \mathbf{p}$ & qjpg & pqjp & py q j & qpjg & g p j q \\
\hline $2 S$ & $\mathrm{XXXX}$ & q j̣pg & pqjp & $\mathbf{X X X X}$ & $\mathbf{X X X X}$ & $\mathbf{X X X X}$ \\
\hline \multicolumn{7}{|l|}{ Dissimilar } \\
\hline $\mathbf{N W}$ & wvsc & $\operatorname{scvw}$ & vwcs & sywv & vwsc & vvwc \\
\hline $2 S$ & $\mathbf{X X X X}$ & $\operatorname{sctvw}$ & rwcs & $\mathbf{X X X X}$ & $\mathbf{X X X X}$ & $\mathbf{X x \times x}$ \\
\hline
\end{tabular}

Note-Examples from Experiment 1 include two consecutive fixations (marked by the dot) for each window size. Examples from Experiment 2 include visually similar and dissimilar backgrounds. In all examples, the target is the letter $y$, which is not present in the Experiment 1 examples but is in the Experiment 2 examples. 
ments were recorded from the right eye, although viewing was binocular. Luminance on the CRT was adjusted to a comfortable level for the subjects, and the subjective brightness was held constant throughout the experiment. The room was dark, except for a dim indirect light source. More details about the characteristics of the apparatus are described by Rayner et al. $(1981,1982)$.

\section{GENERAL RESULTS}

In the experiments to be described, we controlled both the number of characters that were available for processing on each fixation and the background characteristics of the distractor letters (Experiments 2 and 3). Details of the specific experiments are discussed in subsequent sections. Here, we discuss some general findings across the experiments that should be noted, but which are of only tangential interest to our present concerns.

As indicated above, subjects were asked to search for either the letter $b$ or the letter $y$. Across the three experiments, the main effect of target letter was nonsignificant ( $F<1$ in all cases). The specific target letter did occasionally enter into an interaction with another variable in an experiment. However, since there was no consistent pattern across the three experiments, we will not discuss the target letters separately.

The primary dependent variable used in the three experiments was search time expressed as processing time per character (in milliseconds). Thus, total search time was divided by 24 in the target-absent condition and by the serial position (counting from left to right) of the target letter (since subjects were instructed to search from left to right) in the target-present condition. ${ }^{1}$ A comparison of the search times for positive (target-present) and negative (target-absent) trials revealed no differences $(F<1)$. However, there was considerably more variability in search times for positive trials than for negative trials, due primarily to the location of the target. Hence, search times for trials in which the target was absent were used in the data analyses to be reported. (See Jacobs, 1986, for a further rationale for using target-absent lines for data analyses.)

Although search time computed as processing time per character did not differ between positive and negative trials, the total amount of time needed to find the target in the positive trials very much depended on the location of the target within the array. The correlation between the total search time and the location of the target in the array (counting from left to right) was highly significant; the correlations ranged from .77 to .93 across subjects and experiments. This result simply verifies that (1) subjects searched the array from left to right, and (2) the closer to the beginning of the array the target was located, the sooner the subject located it.

Errors in the experiments were very infrequent: The error rate never exceeded more than $1 \%$ of the trials in the first two experiments and was $2.3 \%$ in Experiment 3. The errors were about equally divided between misses and false alarms. The error rates were low presumably because the subjects were well practiced in the experiments.
There was also no indication of any speed-accuracy tradeoffs in the data. The subjects were instructed to search as rapidly as they could without making any errors. The resulting data clearly indicated that they were able to do this.

Finally, it should be noted that in other experiments (Rayner \& Fisher, 1987), we established that the total region from which subjects are able to obtain information in the task is closely comparable to the size of the effective visual field in reading (see Rayner, 1984). As in reading (Rayner \& Fisher, 1987), the perceptual span in the search task was asymmetric, with more information acquired to the right of fixation than to the left; as in reading, subjects in the search task acquired information from 3-4 characters to the left of fixation to about 15 character positions to the right of fixation. ${ }^{2}$ Filling in of the spaces between strings caused considerable disruption in search. However, as in reading (Pollatsek \& Rayner, 1982), much of the disruption that occurred when parafoveal spacing was filled was apparently due to a disruption of the mechanisms involved in determining where to look next. In short, the results reported by Rayner and Fisher (1987) provide some justification both for preserving the spaces between strings and for our use of asymmetric windows in presenting letter information.

\section{EXPERIMENT 1}

In Experiment 1, subjects searched for the target letter in a background of letters selected randomly from the English alphabet (exclusive of $b, y$, or $x$ ). Both window size and string size were varied. By varying the window size, we hoped to determine the size of the region of effective processing. To make this determination, we needed to assume that overall processing efficiency was not affected by the absence of letters outside the region of effective processing. This assumption implies that when the window region contains as many letters as can efficiently be processed on each fixation, the search time will not differ from that of a control condition in which the entire array is available. An example can make the logic clearer. Suppose that the search time when 12 letters appear in the window (say two strings of size 6 each) is no different from the search time in the no-window condition but is faster than the search time when only 6 letters appear in the window. It seems reasonable, then, to conclude that the region of effective processing contains a minimum of 7 letters (the 6 letters in the string currently being fixated plus at least 1 letter in the very next string) and a maximum of 12 letters (the 6 letters in the string currently being fixated plus all letters in the very next string). ${ }^{3}$

We wanted to determine not only the size of the region of effective processing, but also the size of the decision region. Again, the decision region is the area to the left and right of fixation within which subjects are willing to indicate that a target is or is not present. Two things should be noted about this region. First, the boundaries of the region are determined to some extent by the task: if er- 
rors are heavily penalized, then the area will be relatively small; if errors are only lightly penalized, then the area will be relatively large. Second, except for the first fixation, the information used to determine the presence of a target within the decision region will include information from the preview region of the previous fixation. Thus, the size of this region may well be influenced not only by information from the current fixation, but also by information from the immediately previous fixation.

Given the above, the most obvious choice for a measure of the size of the decision region is the saccade size (measured in terms of the number of characters traversed in the average saccade). To visualize this, assume that $l$ letters (to the left of fixation) are in the decision region and that $r$ letters (to the right of fixation) are in the decision region. Then, the decision region contains $l+1+$ $r$ letters. On the next fixation, the subject will presumably move the eyes to the point where again $l$ letters are to the left of fixation. This implies a move, or saccade, of $r+l+1$ letters to the right, a move of the same size as the decision region. ${ }^{4}$

Finally, we wanted to determine the size of the preview region. This size is simply the difference between the size of the region of effective processing and the size of the decision region. Note that the preview region can be as small as size 0 (i.e., nonexistent). There is nothing about the manner in which the sizes of the decision region and the region of effective processing are computed that requires the existence of a preview region.

\section{Method}

There were 32 conditions in the experiment formed by the crossing of four string sizes $(2,4,6$, and 8 letters per string), four window sizes (1S, 2S, 3S, and no window [NW]), and two types of response (target present and target absent). Subjects searched through four blocks of 128 trials. Each block lasted 30-45 min, and the subjects completed one block per day. For two of the blocks, the target letter was $b$, and for the other two, it was $y$. String size and window size were counterbalanced so that an equal number of trials occurred in each condition. Likewise, the frequency of target occurrence was balanced so that targets were present and absent equally often in each of the experimental conditions. As noted above, data from the target-absent trials served as the data for the various analyses that were carried out.

Six subjects served in the experiment. After completing the $\mathbf{4 0}$ practice trials, each subject searched for the target letter through four blocks of 128 trials. Half of the subjects searched for the target letter $b$ for the first 256 trials and then for the target letter $y$ on the remaining half of the trials; the other half of the subjects searched for $y$ first then $b$. All of the letters outside the window were replaced by Xs, as shown in Table 1 .

\section{Results and Discussion}

As indicated previously, search time was the primary dependent variable. However, eye-movement behavior was also examined. The number and mean lengths of leftto-right and right-to-left saccades were computed for each subject, as were the durations of fixations. However, since the subjects searched the array from left to right, there were very few right-to-left saccades. In fact, right-to-left saccades constituted only $8 \%$ of the total number of sac- cades, and a high proportion of these occurred in the conditions in which both string size and window size were small. Since the average durations of fixations following left-to-right saccades and right-to-left saccades did not differ much, and since some of the subjects made very few right-to-left saccades at all, we collapsed across the direction of the saccade in most of the analyses to be reported. ${ }^{5}$ The major exception is that we do report the length of left-to-right saccades (note that between-string spaces were counted in the computation of saccade length). We will discuss the search time first. Following that we will present the eye-movement data.

Search time. Figure 1 shows the search time for the different conditions in Experiment 1. Analyses of variance revealed that there were significant main effects of window size $[F(3,15)=31.18, p<.001]$ and string size $[F(3,15)=388.21, p<.001]$, as well as an interaction of two $[F(9,45)=23.36, p<.001]$. With respect to the main effect of string size, search was slower when the stimulus array consisted of a string size of 2 than when it consisted of a string size of $4(p<.01)$. In turn, search when string size was 4 was slower $(p<.01)$ than when the size was 6 or 8 . However, search was no slower when the string size was 6 than when it was 8 . If string size 2 is excluded, it is clear that for all string sizes search time reached asymptote when the string to the right of fixation was available. Indeed, the interaction is primarily due to the fact that all other string sizes were at asymptote when the string to the right of fixation was available, whereas for string size 2 search time continued to improve as window size increased.

An estimate of the region of effective letter processing can be obtained by examining search time solely as a function of the number of letters from the array that were available. This procedure involves collapsing across window size and string size. However, when we did this, it was very clear that the spacing between strings played a powerful role in determining overall performance levels. Thus, for example, when 6 letters were available, search time when the 6 letters resulted from string size 2 with a window size of $3 \mathrm{~S}$ was not the same as when the string size was 6 and the window size was 1S. In Table 2 we have made that distinction. A perusal of Table 2 and Figure 1 indicates that at least 9 characters are processed in each fixation. There is a nice consistency across string sizes in this estimate. For example, note that with a string size of 4 , asymptote is reached with a window size of 3 strings (indicating that at least 9 characters are processed in each fixation), and with a string size of 8 , asymptote is reached with a window size of 2 strings (indicating again that at least 9 characters are processed in each fixation).

Although the estimate of 9 letters might be a reasonable approximation of how many letters at a minimum receive some type of processing, we believe that it would be a mistake to conclude that subjects determined with complete assurance whether each of the 9 letters was or was not a target. In particular, the eye-movement data suggest that no more than 4 letters were in the decision region. We turn now to those data. 


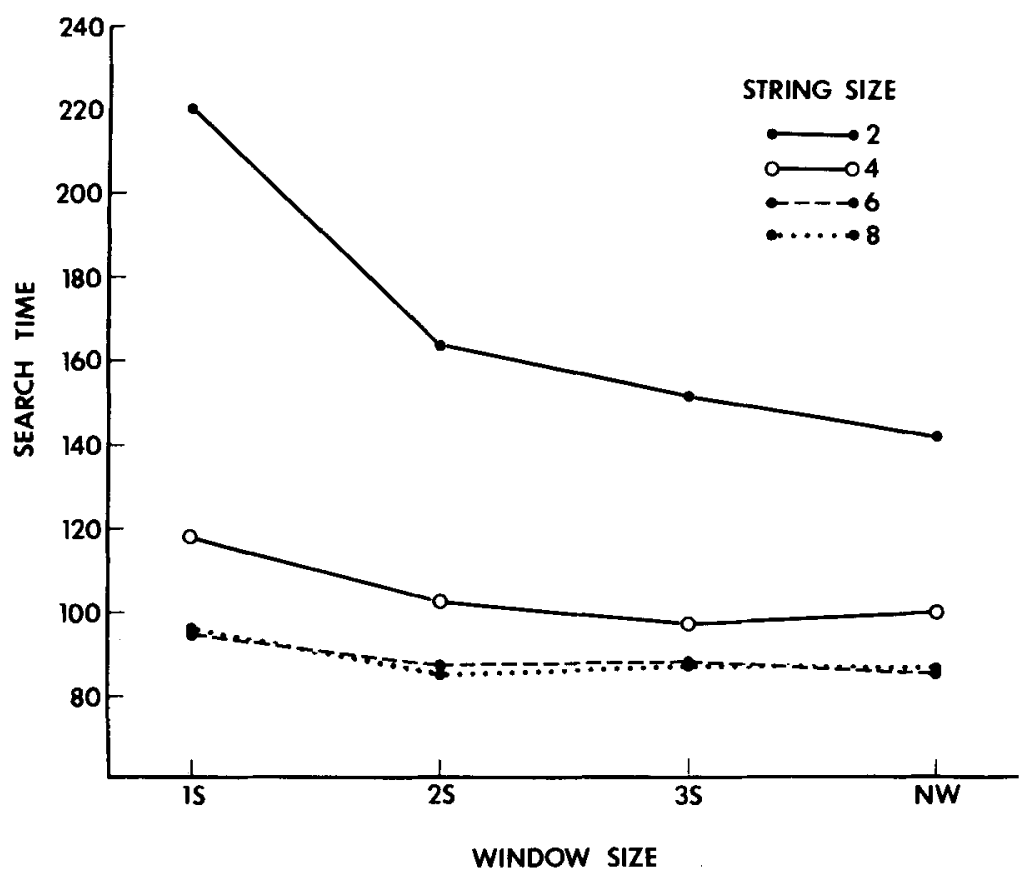

Figure 1. Search time as a function of string size and window size in Experiment 1.

Eye-movement data. The eye-movement data from the experiment are presented in Tables 3-5. The total number of fixations, fixation duration, and saccade length were all affected by the window size (all $F_{\mathrm{s}}>8.14, p<.01$ ) and string size (all $F \mathrm{~s}>5.89, p<.05$ ).

The number of fixations that subjects made followed a pattern quite similar to the search time data, with the interaction of window size and string size being significant $[F(9,45)=3.8, p<.01]$. Likewise, for fixation duration, the interaction was significant $[F(9,45)=6.07$, $p<.01]$. However, it is clear that there was little in the way of a difference in fixation duration for string sizes of 6 and 8, irrespective of window size. For saccade length, the interaction of window size and string size was nonsignificant $(F<1)$.

If we make the assumption that saccade length reflects the number of letters in the decision region (see above), we would estimate that 3-4 letters were in this region in normal search (see NW condition, Table 5). Note that, in terms of how far they moved their eyes, the subjects were strongly influenced by the length of the string. In particular, the results suggest that shorter strings compress the length of the saccade. This is especially apparent for strings of length 2 at all window sizes. However, in all cases, the subjects tended to move to the immediately adjacent string, seldom skipping over a string. Other researchers (Engel, 1977; Levy-Schoen, 1974) have reported that the tendency to fixate the object closest to fixation is very coercive, and our data appear to agree with this conclusion.

In summary, on the basis of Experiment 1, we would want to conclude that at least 9 letters received some type of processing in each fixation, but that the decision region consists of 3-4 letters per fixation.

If saccade size is, indeed, a measure of the size of the decision region, then saccade size should be larger with

Table 2

Mean Search Time (in Milliseconds) in Experiment 1 as a Function of the Number of Characters Available in the Array

\begin{tabular}{|c|c|c|c|c|c|c|c|c|c|c|c|c|}
\hline $\begin{array}{l}\text { Characters } \\
\text { Available: }\end{array}$ & 2 & 4 & 4 & 6 & 6 & 8 & 8 & 12 & 12 & 16 & 18 & 24 \\
\hline Window Size: & is & $2 S$ & is & $3 S$ & $1 S$ & $2 S$ & is & $3 S$ & $2 S$ & $2 S$ & $3 S$ & $3 S$ \\
\hline String Size: & 2 & 2 & 4 & 2 & 6 & 4 & 8 & 4 & 6 & 8 & 6 & 8 \\
\hline $\begin{array}{l}\text { Search Time: } \\
\text { Mean Time: }\end{array}$ & 220 & & & & & & & & & $85 *$ & 88* & $87 *$ \\
\hline
\end{tabular}

Note-Row labeled Search Time includes the means when a given number of letters could be available in two ways: for example, 4 letters could be available with string size 2 and window size $2 S$ (164 msec per character) or with string size 4 and window size 15 (118 msec per character). Row labeled Mean Time is the mean for the number of letters available. The asterisks indicate asymptotic performance and conditions that do not differ from asymptote $(p<.05)$. 
Table 3

Mean Fixation Duration (in Milliseconds) in Experiment 1 as a Function of Window Size and String Size

\begin{tabular}{ccccc}
\hline \multicolumn{3}{c}{ as a Function of Window Size and String Size } \\
\cline { 2 - 5 } Window Size & \multicolumn{4}{c}{ String Size } \\
\cline { 2 - 5 } & $\mathbf{2}$ & 4 & $\mathbf{6}$ & $\mathbf{8}$ \\
\hline 1S & 317 & 297 & 264 & 260 \\
3S & 269 & 272 & 267 & 261 \\
NW & 263 & 274 & 263 & 266 \\
NS & 254 & 274 & 258 & 263 \\
\hline
\end{tabular}

Table 4

Total Number of Fixations in Experiment 1 as a Function of Window Size and String Size

\begin{tabular}{ccccc}
\hline & \multicolumn{4}{c}{ String Size } \\
\cline { 2 - 5 } Window Size & 2 & 4 & 6 & 8 \\
\hline IS & 16.7 & 9.6 & 8.5 & 8.8 \\
2S & 14.2 & 8.9 & 6.8 & 7.0 \\
3S & 13.1 & 8.4 & 6.9 & 7.1 \\
NW & 12.9 & 8.3 & 6.8 & 7.0 \\
\hline
\end{tabular}

a background of visually dissimilar distractors than with a background of visually similar distractors. Furthermore, if more than just location information (i.e., information about where next to place the eyes) is gathered from the preview region, then there should be a further expansion of this region into the parafovea. This conclusion is based on the fact that location information does not vary with changes in the similarity of the targets to the distractors. Thus, any change in the extent of this region with changes in the target-distractor similarity must be explained by factors other than location information. Note that since acuity drops off precipitously in the preview region (i.e., in the parafovea), one might expect to find that only location information was actually obtainable from this region.

Neisser $(1963,1964)$, among others, has looked at search performance as a function of target-distractor similarity. He demonstrated that subjects could search faster through arrays in which the background distractor items were visually dissimilar to the target. However, Neisser did not show what effect the change in targetdistractor similarity had on the extent of the decision and preview regions.

\section{EXPERIMENT 2}

In Experiment 2, subjects searched for a target letter in arrays made up of visually similar or visually dissimilar letters within the window. Letters outside the window were replaced by Xs. String size and window size were varied. By varying the string and window sizes and by recording eye movements, we hoped to determine the influence of background characteristics on the sizes of the decision and preview regions. In addition, we hoped to determine whether information obtained in the preview region was confined to details about spatial location for upcoming eye movements.

\section{Method}

In this experiment, there were 64 conditions formed by the crossing of four string sizes $(2,4,6$, and 8 letters per string), four window sizes (1S, $2 S, 3 S$, and NW), two types of response (target present and target absent), and two types of distractor letters (similar and dissimilar). The subjects searched through eight blocks of 128 trials. Each block lasted approximately 30-45 min, and the subjects completed two blocks per day (with a rest period between the two blocks). Each session was preceded by $\mathbf{4 0}$ practice trials. The target letter was $b$ for half the blocks and $y$ for the other half. When $b$ was the target letter, the visually similar arrays consisted of the letters $h, k, d$, and $l$. When $y$ was the target letter, the visually similar arrays consisted of the letters $g, j, q$, and $p$. Dissimilar arrays consisted of the letters $v, s, c, z, r, w$, and $n$.

Four of the subjects from Experiment 1 participated in this experiment. After completing the practice trials, each subject searched for the target letter through blocks of 128 trials. Half of the subjects searched for the target letter $b$ for the first half of the trials; the other half searched for the $y$ first. All of the variables were counterbalanced so that an equal number of trials occurred for each condition.

\section{Results and Discussion}

Search time. Figure 2 shows the search times for the different conditions in Experiment 2. As in Experiment 1, there were significant main effects of window size $[F(3,9)$ $=41.09, p<.001]$ and string size $[F(3,9)=292.24$, $p<.001]$, as well as an interaction of the two $[F(9,27)$ $=32.09, p<.001]$. As can be seen in Figure 2, the data basically replicate Experiment 1 with respect to the interaction. For all conditions except string size 2, performance reached asymptote when the string to the right of the currently fixated string was available; for string size 2 , search performance increased as the window became larger.

We again collapsed across conditions to obtain an estimate of the number of letters processed per fixation. Again, the results were highly similar to those obtained in Experiment 1 (see Table 6). Examination of this table and Figure 2 reveals that when the distractors were similar to the target, in the best of circumstances at least 9 letters received some type of processing. However, when the distractors were dissimilar to the target, in the best of circumstances at least 13 letters received some processing (see string size 6). Thus, the extent of the region of effective processing increased considerably when the background consisted of dissimilar distractors.

As in Experiment 1, there was a large difference in the search times for string sizes 4 and 6 , but little difference in the search times for string sizes 6 and 8 . These rela-

Table 5

Mean Saccade Length (in Character Spaces) in Experiment 1 as a Function of Window Size and String Size

\begin{tabular}{ccccc}
\hline & \multicolumn{4}{c}{ String Size } \\
\cline { 2 - 5 } Window Size & 2 & 4 & 6 & 8 \\
\hline 1S & 2.5 & 3.0 & 3.0 & 3.1 \\
2S & 2.8 & 3.2 & 3.5 & 3.5 \\
3S & 2.9 & 3.3 & 3.4 & 3.4 \\
NW & 3.0 & 3.5 & 3.5 & 3.5 \\
\hline
\end{tabular}




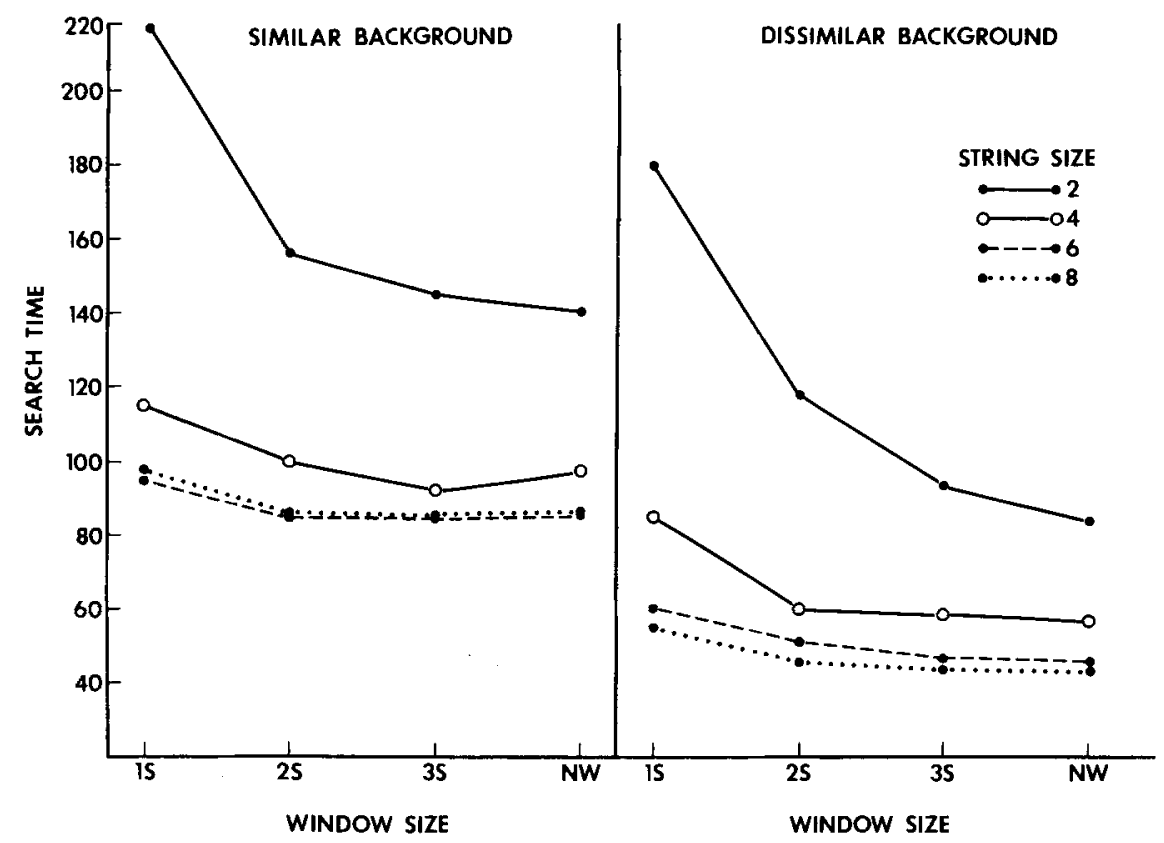

Figure 2. Search time as a function of string size and window size in Experiment 2. The left panel is for similar backgrounds and the right panel is for dissimilar backgrounds.

tionships hold for both the visually similar and the visually dissimilar arrays.

The major difference between Experiment 1 and the present experiment was that in the present experiment we varied the characteristics of the background distractor items. As expected, this manipulation had a major effect on search time, since subjects searched much faster when the background consisted of dissimilar letters $(70 \mathrm{msec}$ per character collapsed across conditions) than when it consisted of similar letters (111 msec per character) $[F(1,3)=223.42, p<.01\}$. However, background similarity did not interact with any other variable, as is clear in Figure 2.

Eye-movement data. The eye-movement data from the experiment are shown in Tables 7-9. As in Experiment 1, the total number of fixation, fixation durations, and sac- cade lengths were all affected by window size (all $F$ s $>9.92, p<.01$ ) and string size (all $F$ s $>4.67$, $p<.05$ ). The interaction for two of the dependent variables was significant $[F(9,27)=8.23$ and $4.31, p<.01$, for number of fixations and fixation duration, respectively]. As in Experiment 1, there was little in the way of differences in fixation duration for string sizes of 6 and 8 irrespective of window size. Similar distractors resulted in more fixations (9.7) than did dissimilar distractors (6.8) $[F(1,3)=161.1, p<.01]$ and in longer fixation durations $(275 \mathrm{msec}$ for similar distractors and 239 for dissimilar distractors) $[F(1,3)=49.84, p<.01]$, but in both cases background characteristics did not interact with any other variable.

For saccade length, again as in Experiment 1, the interaction of window size and string size was not signifi-

Table 6

Search Time (in Milliseconds) in Experiment 2 as a Function of the Number of Letters Available in the Array

\begin{tabular}{|c|c|c|c|c|c|c|c|c|c|c|c|c|}
\hline \\
\hline Available: & 2 & 4 & 4 & 6 & 6 & 8 & 8 & 12 & 12 & 16 & 18 & 24 \\
\hline Window Size: & $1 S$ & $2 S$ & $1 S$ & $3 S$ & $1 S$ & $2 S$ & 1S & $3 S$ & $2 S$ & $2 S$ & 3S & $3 S$ \\
\hline String Size: & 2 & 2 & 4 & 2 & 6 & 4 & 8 & 4 & 6 & 8 & 6 & \\
\hline \multicolumn{13}{|c|}{ Similar Background } \\
\hline Search Time: & & 156 & & 145 & & 100 & & 96 & & & & \\
\hline Mean Time: & 217 & & & & & & & & & $86^{*}$ & $86 *$ & $86^{*}$ \\
\hline \multicolumn{13}{|c|}{ Dissimilar Background } \\
\hline Search Time: & & 118 & & 93 & & 61 & & & 50 & & & \\
\hline Mean Time: & 180 & & & & & & & & & $42^{*}$ & $44 *$ & $43 *$ \\
\hline
\end{tabular}

Note-Rows labeled Search Time include the means for a given number of letters computed in two ways. Rows labeled Mean Time include the means for the number of letters available. The asterisks indicate asymptotic performance and conditions that do not differ from asymptote $(p<.05)$. 
Table 7

Mean Fixation Duration (in Milliseconds) in Experiment 2 as a Function of Window Size, String Size, and Background Similarity

\begin{tabular}{ccccc}
\hline & \multicolumn{4}{c}{ String Size } \\
\cline { 2 - 5 } Window Size & 2 & 4 & 6 & 8 \\
\hline \multicolumn{5}{c}{ Similar Background } \\
IS & 298 & 270 & 258 & 248 \\
3S & 295 & 276 & 285 & 307 \\
NW & 276 & 274 & 265 & 265 \\
& 263 & 268 & 264 & 274 \\
IS & Dissimilar & Background & \\
2S & 298 & 230 & 232 & 234 \\
3S & 273 & 244 & 240 & 231 \\
NW & 250 & 229 & 236 & 212 \\
\end{tabular}

Table 8

Total Number of Fixations in Experiment 2 as a Function of Window Size, String Size, and Background Similarity

\begin{tabular}{|c|c|c|c|c|}
\hline \multirow[b]{2}{*}{ Window Size } & \multicolumn{4}{|c|}{ String Size } \\
\hline & 2 & 4 & 6 & 8 \\
\hline \multicolumn{5}{|c|}{ Similar Background } \\
\hline is & 17.8 & 9.3 & 8.4 & 8.8 \\
\hline $2 S$ & 13.7 & 8.7 & 7.6 & 7.7 \\
\hline $3 S$ & 13.2 & 8.1 & 7.8 & 7.9 \\
\hline NW & 12.7 & 7.7 & 7.8 & 7.7 \\
\hline \multicolumn{5}{|c|}{ Dissimilar Background } \\
\hline $1 \mathrm{~S}$ & 14.5 & 7.5 & 5.9 & 5.2 \\
\hline $2 S$ & 12.0 & 6.0 & 5.1 & 4.4 \\
\hline $3 S$ & 9.3 & 5.8 & 4.8 & 4.6 \\
\hline NW & 8.2 & 5.8 & 4.7 & 4.3 \\
\hline
\end{tabular}

cant $[F(9,27)=1.99, p>.08]$. Similar letters in the background decreased saccade length ( 3.25 characters) relative to dissimilar letters in the background (4.6 characters) $[F(1,3)=18.04, p<.05]$. However, unlike the other two dependent variables, with saccade length background characteristics interacted with both window size $[F(3,9)=6.42, p<.05]$ and string size $[F(3,9)=$ $14.37, p<.01]$. In the former case, the interaction was due to little increase in saccade length as window size increased when the background letters were similar to the target, coupled with a steady increase in saccade length as window size increased when the background letters were dissimilar to the target. In the latter case, the interaction was likewise due to little increase in saccade length as string size increased with similar letters contrasted with an increase in saccade length as string size increased (up to string size 6 , which did not differ from size 8) with dissimilar letters. The three-way interaction of these variables was not significant $[F(3,27)=1.97, p>.08]$.

If we again assume that saccade length reflects the number of letters in the decision region, we would estimate that 3-6 letters are in the region per fixation. As shown in Table 9, fewer letters were in the region when the distractors were similar to the target (about 3-4 letters) than when they were dissimilar (5-6 letters). As the interac- tions we have described document, how far the eyes moved on average was influenced by the window size, the string size, and the background characteristics.

The results above on both saccade size and the time to process each letter indicate that the decrease in search time that was observed in the dissimilar arrays is the product of two factors: an increase in both the number of letters processed in a single fixation and the speed of letter processing. This is to be expected if the time to process each letter is related to the number of features that must be processed: as the distractors become more dissimilar to the target, the number of features that overlap with the target decreases. Furthermore, as fewer features are needed to analyze the target, the target can be identified farther out in the parafovea.

In conclusion, the major finding of Experiment 2 was that the similarity of the target to the background distractor items strongly influenced search performance. Specifically, the extent of both the decision and preview regions (and therefore the region of effective processing) increased in the dissimilar condition. The fact that the decision region increases in size is not at all unexpected and is certainly consistent with a great deal of other research. The fact that the preview region extends farther into the parafovea, while not totally unexpected, is of some note, since this increase indicates that more than just location information is being obtained from the preview region.

The question that remains is: What information in addition to location information is being processed in the preview region? One initially likely candidate might appear to be information about the absence of a target. However, if information about the absence of a target were indeed processed, subjects should logically be able to extend the decision region into the preview region. Clearly, this was not the case, since the preview region extends considerably beyond the decision region. A second likely candidate is partial letter information. This could be either a recording of a subset of the features or a preliminary activation of an abstract letter code (Rayner, 1984). Experiment 3 was an attempt to determine whether, in addition to location information, such partial letter information was obtained in the parafovea.

Table 9

Mean Saccade Length (in Character Spaces) in Experiment 2 as a Function of Window Size, String Size, and Background Similarity

\begin{tabular}{|c|c|c|c|c|}
\hline \multirow[b]{2}{*}{ Window Size } & \multicolumn{4}{|c|}{ String Size } \\
\hline & 2 & 4 & 6 & 8 \\
\hline \multicolumn{5}{|c|}{ Similar Background } \\
\hline $\begin{array}{c}1 S \\
2 S \\
3 S \\
\text { NW }\end{array}$ & $\begin{array}{l}2.8 \\
2.9 \\
3.0 \\
2.9\end{array}$ & $\begin{array}{l}3.0 \\
3.4 \\
3.4 \\
3.6\end{array}$ & $\begin{array}{l}3.2 \\
3.6 \\
3.4 \\
3.5\end{array}$ & $\begin{array}{l}3.1 \\
3.5 \\
3.5 \\
3.5\end{array}$ \\
\hline \multicolumn{5}{|c|}{ Dissimilar Background } \\
\hline $\begin{array}{c}1 S \\
2 S \\
3 S \\
\text { NW }\end{array}$ & $\begin{array}{l}2.8 \\
3.1 \\
4.2 \\
4.6\end{array}$ & $\begin{array}{l}3.9 \\
4.7 \\
4.7 \\
4.9\end{array}$ & $\begin{array}{l}4.5 \\
4.9 \\
5.1 \\
5.4\end{array}$ & $\begin{array}{l}4.8 \\
5.2 \\
5.1 \\
5.6\end{array}$ \\
\hline
\end{tabular}




\section{EXPERIMENT 3}

In Experiment 3, subjects searched for a target letter that (when present) was always in the array regardless of whether it was inside or outside the window. There were four primary conditions, in which $\mathrm{W}$ refers to letters within the window, $O$ refers to letters outside the window, $S$ refers to distractors similar to the target, and D refers to distractors dissimilar to the target: WS-OS, in which the background letters inside and outside the window were visually similar to the target; WD-OD, in which the background letters inside and outside the window were visually dissimilar to the target; WS-OD, in which background letters inside the window were visually similar to the target while those outside the window were dissimilar to the target; WD-OS, in which the background letters inside the window were dissimilar to the target while those outside the window were visually similar to the target.

Note that there are two important differences between this experiment and Experiments 1 and 2. First, in this experiment, the letters outside the window (exclusive of the target) were replaced by other letters, which were visually similar or dissimilar to the target, rather than by $\mathrm{Xs}$ as in Experiments 1 and 2. Thus, in Experiment 3, the boundary of the window was not distinct and subjects could easily process erroneous information (i.e., letters that changed their identity when fixated). A second important difference between Experiment 3 and the first two experiments is that, in Experiment 3 the target was never replaced by another letter when it was outside the window; in the first two experiments the target was replaced by an $\mathrm{X}$ when it was outside the window. Thus, in Experiment 3 , subjects could potentially identify the target when it was outside the window.

The above manipulations allowed us to determine whether partial letter information was obtained from the parafoveal preview region. Note that Condition WS-OS in Experiment 3 is identical to the NW similar condition of Experiment 2 in all respects but one: in Experiment 3, the distractor letters in the parafovea changed their identity (but not their similarity to the target) when they were foveated. Thus, if letter information were being acquired from the parafovea, there should be evidence of a longer mean fixation duration in Condition WS-OS in Experiment 3 than in the NW similar condition of Experiment 2. The argument is straightforward: If it is at all effective, the partial letter information obtained in the parafovea on fixation $n$ should speed processing on fixation $n+1$ if the letters remain the same; likewise, a change in letter information should slow processing. A similar increase in the mean fixation duration should be noted for Condition WD-OD in Experiment 3 when compared with the dissimilar condition in Experiment 2.

There is a related test for partial letter information which should be mentioned. Briefly, if subjects derive partial letter information from the parafovea, then there should be a decrease in the time to process each character as the window size increases for both Condition WS-OS and Condition WD-OD. This is because, at the larger window sizes, the letters seen in the decision region on fixation $n+1$ are more likely to be exact copies of the letters seen in the parafoveal preview region on fixation $n$. Thus, whatever influence partial letter information has on processing within the decision region should increase as the window size increases.

\section{Method}

There were 128 conditions in the experiment formed by the crossing of four string sizes $(2,4,6$, and 8 letters per string), four window sizes (1S, $2 \mathrm{~S}, 3 \mathrm{~S}$, and no window), two types of response (target present and target absent), and four types of background conditions. In the WS-OS condition, the letters inside the window were visually similar to the target (when $b$ was the target, the distractor letters were $h, k, d$, and $l$ ) and the letters outside of the window area were also visually similar to the target. In the WD-OD condition, letters inside the window were visually dissimilar to the target (when $b$ was the target, the distractor letters were $r, v, z$, and $n$ ) and the letters outside the window were also visually dissimilar. In the WSOD condition, letters inside the window were visually similar to the target but the letters outside the window were replaced by visually dissimilar letters. Finally, in the WD-OS condition, letters inside the window were visually dissimilar to the target but the letters outside the window were replaced by visually similar letters. Table 10 shows an example of each of the four conditions. Note that even in the WS-OS and WD-OD conditions, letters from the original string (i.e., the string in the $\mathrm{NW}$ condition) outside the window were replaced by other letters (except for the target when it was present).

Subjects searched through 16 blocks of 128 trials. Each block lasted 35-45 min, and subjects completed two to four blocks per day (with rest periods between the blocks). For half of the blocks, the target letter was $b$; for the other half it was $y$. Half of the subjects searched for the target letter $b$ for the first half of the trials; the other half searched for $y$ first. All of the variables were counterbalanced so that an equal number of trials occurred for each condition. At the beginning of each session, 40 practice trials were presented. The 4 subjects from Experiment 2 participated in this experiment.

\section{Results and Discussion}

Search time. Figure 3 shows the search times for the different conditions in Experiment 3. As in Experiments 1 and 2, there were significant main effects of window size $[F(3,9)=12.72, p<.01]$ and string size $[F(3,9)$ $=23.46, p<.001]$. Unlike the first two experiments, the interaction of the two variables was not significant $(p>.20)$. However, window size and string size both

Table 10

Sample Stimulus Arrays from Experiment 3

\begin{tabular}{|c|c|c|c|c|}
\hline \multirow{2}{*}{$\frac{\text { Condition }}{\text { NW }}$} & \multicolumn{4}{|c|}{ Stimulus Arrays } \\
\hline & pqgjgp & $q j p g p g$ & gpy jgp & p j g q p g \\
\hline WS-OS & $\mathbf{j} \mathbf{p q} \mathbf{g} \mathbf{q} \mathbf{j}$ & qjpg pg & $q \mathbf{j}$ y $g \mathrm{qj}$ & $\mathrm{j} g q \mathrm{pj}$ \\
\hline WS-OD & wcsvsw & qj p g p g & swy ysw & wrscws \\
\hline NW & $w v \operatorname{csvc}$ & vcw csc & $\operatorname{csy} \mathrm{w} v \mathrm{c}$ & $\operatorname{scsv} \mathbf{v}$ \\
\hline WD-OD & $\operatorname{csw} v s w$ & v c wesc & wvycsw & vwrscs \\
\hline WD-OS & $\mathrm{pj} \mathrm{q} \mathrm{g} \mathrm{j} \mathrm{q}$ & ve wes c & $q g$ y $p$ j q & $\mathbf{g} q \mathbf{g} \mathbf{j} \mathbf{p} \mathbf{j}$ \\
\hline
\end{tabular}

Note-Examples are all of a $1 S$ window with the fixation location marked by the dot. The target is the letter $y$. 


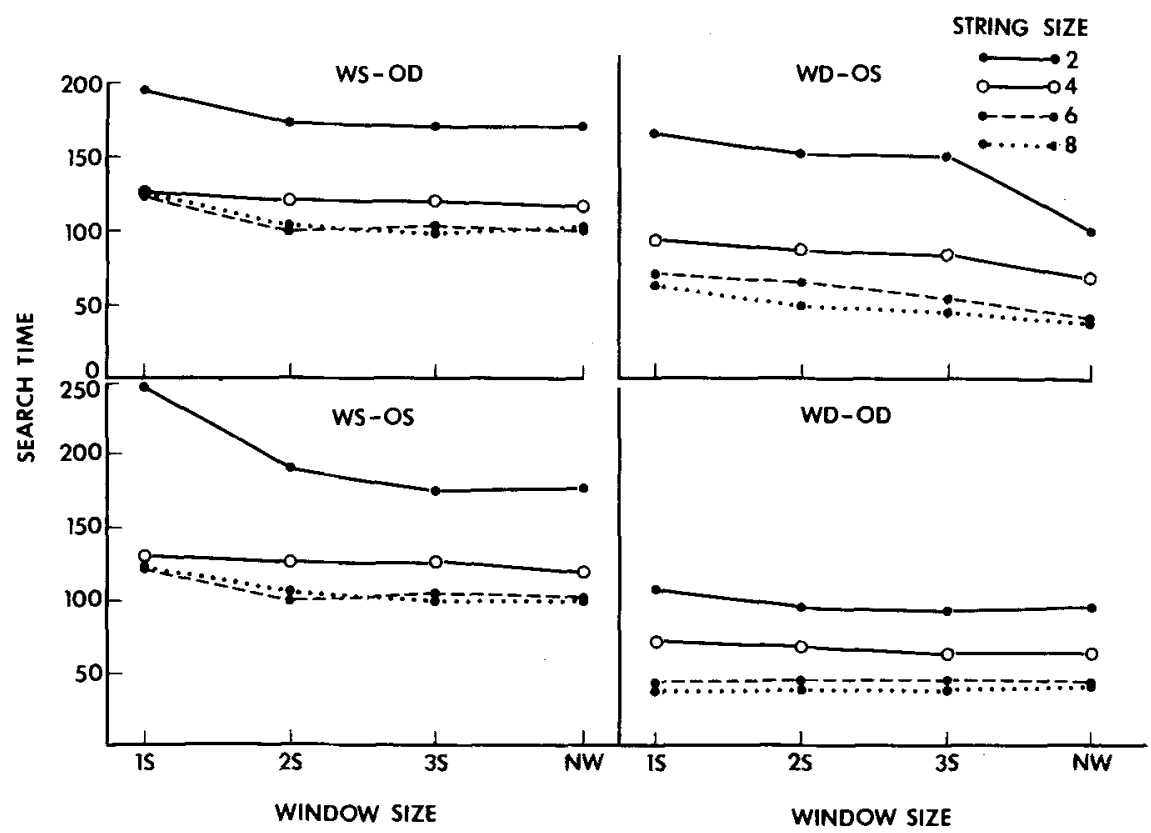

Figure 3. Search time as a function of string size and window size in Experiment 3. Upper left panel, Condition WS-OD; lower left panel, Condition WS-OS; upper right panel, Condition WDOS; and lower right panel, Condition WD-OD.

entered into significant interactions with background characteristics. As in Experiment 2, subjects searched faster when the letters inside the window were dissimilar to the target (73 msec per character) than when they were similar (130 msec per character) to the target $[F(1,3)=$ $69.22, p<.01]$. Likewise, when the letters outside the window were dissimilar to the target, subjects searched faster ( $95 \mathrm{msec}$ per character) than when they were similar (108 msec per character) $[F(1,3)=34.19, p<.05]$. The following interactions involving background characteristics were significant: window size $\times$ parafoveal background $[F(3,9)=7.51, p<.01]$, string size $\times$ parafoveal background $[F(3,9)=8.78, p<.01]$, window size $\times$ parafoveal background $\times$ foveal background $[F(3,9)$ $=6.81, p<.05]$, window size $\times$ string size $\times$ parafoveal background $[F(9,27)=4.78, p<.001]$, string size $\times$ parafoveal background $\times$ foveal background $[F(3,9)$ $=5.73, p<.05]$, and the four-way interaction $[F(3,27)$ $=2.98, p<.05]$.

The information in Figure 3 and Table 11 can be used to determine whether partial letter information is obtained from the preview region in Conditions WS-OS and WDOD. Note that in Condition WS-OS, search time decreases as the window size increases from $1 S$ to $2 S$. Thus, it is reasonable to conclude that some letter information is being gathered from the preview region. However, note that in Condition WD-OD there was considerably less of a decrease in search time. Furthermore, this decrease was confined primarily to strings of 2 letters each. Thus, it appears that partial letter information is much less important in Condition WD-OD than in Condition WS-OS. This is exactly what we would expect if subjects were processing only at the featural level in the dissimilar condition, an expectation suggested by the results from Experiment 2.

The above results can also be used to provide additional evidence that a decision is not made about the absence of a target in the preview region. If such a decision were made, then one would predict an increase in search time with an increase in the window size for Condition WSOD. This will be the case because, at the smaller window sizes, subjects will need to process fewer similar letters in the preview region. If they can skip over the letters in the preview region when the target is absent, then the above prediction follows immediately. However, as Figure 3 makes clear, such an increase was not observed.

Eye-movement data. The eye-movement data are shown in Tables 12-14. For the total number of fixations, there was a main effect of window size $[F(3,9)=5.34$, $p<.05]$ and a marginally significant effect of string size $[F(9,27)=1.89, p<.10]$. Subjects made fewer fixations when the letters inside the window were dissimilar to the target (6.55) than when they were similar (10.3) $[F(1,3)=81.82, p<.01]$. They also made fewer fixations when the letters outside the window were dissimilar to the target (8.11) than when they were similar (8.73) $[F(1,3)=46.11, p<.01]$ : Background characteristics also interacted with window size and/or string size. The following interactions were significant: parafoveal background $\times$ string size $[F(3,9)=4.68, p<.05]$, window size $\times$ foveal background $\times$ string size $[F(3,9)=4.68$, $p<.05]$, and window size $\times$ foveal background $\times$ parafoveal background $[F(9,27)=3.88, p<.05]$. The following interactions were marginally significant: win- 
Table 11

Search Time (in Milliseconds) in Experiment 3 as a Function of the Number of Letters Available in the Array

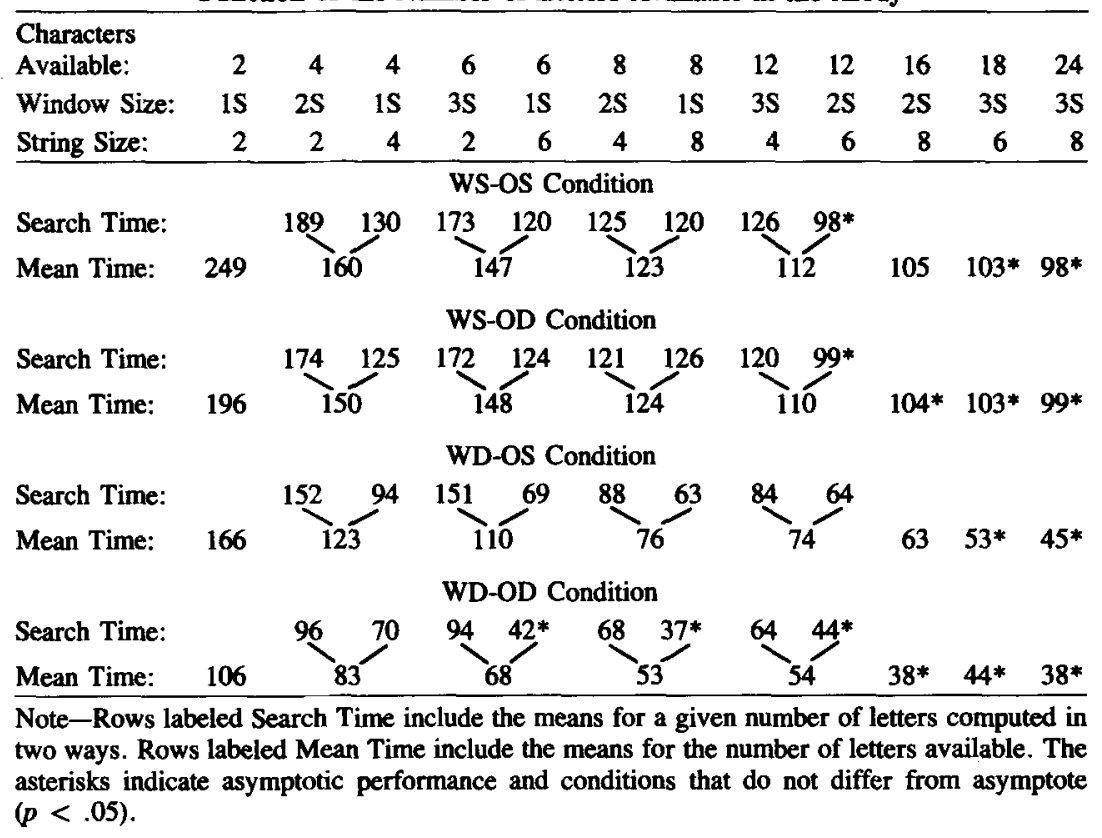

dow size $\times$ string size $\times$ parafoveal background $[F(9,27)$ $=2.07, p<.07]$ and string size $\times$ foveal background $\times$ parafoveal background $[F(3,9)=3.13, p<.08]$.

For fixation duration, there was a significant main effect of window size $[F(3,9)=5.88, p<.05]$. Although fixations seemed to be longer with shorter strings, neither the main effect of string size $(p>.10)$ nor the interaction $(p>.30)$ was significant. Fixation durations were longer when the letters inside the window were similar to the target $(306 \mathrm{msec})$ than when they were dissimilar $(259 \mathrm{msec})[F(1,3)=26.65, p<.05]$; they were also longer when the letters outside the window were similar $(290 \mathrm{msec})$ than when they were dissimilar $(274 \mathrm{msec})$ $[F(1,3)=8.99, p<.06]$. An interaction of foveal background $\times$ parafoveal background $[F(1,3)=14.85$, $p<.05]$ showed that when the letters inside the window were dissimilar, fixation durations were shorter when the letters outside the window were also dissimilar than when they were similar, and that when the letters inside the window were similar it made no difference if the letters outside were similar or dissimilar. This tendency was also more pronounced for smaller window sizes $[F(3,9)=$ 3.33, $p<.07$ ].

For saccade length, there was a significant main effect of string size $[F(3,9)=18.17, p<.001]$. Although saccade length was shorter with smaller window sizes, the main effect did not reach significance $[F(3,9)=2.22$, $p<.10$ ]. Saccade length was longer when the letters inside the window were dissimilar to the target $(4.6$ characters) than when they were similar $(3.16$ characters) $[F(1,3)$ $=21.34, p<.05]$. Saccades were also a bit longer when the letters outside the window were dissimilar (4.01 characters) than when they were similar ( 3.74 characters), but the difference was not significant $[F(1,3)=5.03$, $p<.10]$. The only interaction that approached significance was that of foveal background $X$ string size $[F(3,9)=2.99, p<.09]$.

The above results can be used to determine whether partial letter information is obtained from the parafoveal preview region. We have already noted that the search-

Table 12

Mean Fixation Duration (in Milliseconds) in Experiment 3 as a Function of Window Size, String Size, and Background Similarity

\begin{tabular}{|c|c|c|c|c|}
\hline \multirow[b]{2}{*}{ Window Size } & \multicolumn{4}{|c|}{ String Size } \\
\hline & 2 & 4 & 6 & 8 \\
\hline \multicolumn{5}{|c|}{ WS-OS } \\
\hline 1S & 358 & 348 & 320 & 276 \\
\hline $2 S$ & 322 & 299 & 302 & 308 \\
\hline $3 S$ & 311 & 297 & 290 & 282 \\
\hline NW & 312 & 301 & 296 & 293 \\
\hline \multicolumn{5}{|c|}{ WS-OD } \\
\hline $1 S$ & 309 & 322 & 329 & 281 \\
\hline $2 S$ & 312 & 298 & 302 & 322 \\
\hline 3S & 316 & 303 & 309 & 286 \\
\hline NW & 311 & 292 & 294 & 291 \\
\hline \multicolumn{5}{|c|}{ WD-OS } \\
\hline 1S & 303 & 327 & 284 & 304 \\
\hline $2 S$ & 304 & 298 & 275 & 239 \\
\hline $3 S$ & 312 & 280 & 249 & 244 \\
\hline NW & 265 & 258 & 240 & 224 \\
\hline \multicolumn{5}{|c|}{ WD-OD } \\
\hline 1S & 250 & 275 & 250 & 238 \\
\hline $2 S$ & 256 & 269 & 251 & 209 \\
\hline $3 S$ & 254 & 253 & 244 & 224 \\
\hline NW & 259 & 254 & 223 & 214 \\
\hline
\end{tabular}


Table 13

Total Number of Fixations in Experiment 3 as a Function of Window Size, String Size, and Background Similarity

\begin{tabular}{|c|c|c|c|c|}
\hline \multirow[b]{2}{*}{ Window Size } & \multicolumn{4}{|c|}{ String Size } \\
\hline & 2 & 4 & 6 & 8 \\
\hline \multicolumn{5}{|c|}{ WS-OS } \\
\hline 1S & 16.6 & 8.9 & 9.1 & 10.8 \\
\hline $2 S$ & 14.3 & 10.4 & 8.0 & 8.4 \\
\hline $3 S$ & 14.0 & 10.6 & 7.9 & 8.7 \\
\hline NW & 13.3 & 9.7 & 8.2 & 8.2 \\
\hline \multicolumn{5}{|c|}{ WS-OD } \\
\hline 1S & 15.1 & 9.4 & 9.1 & 10.8 \\
\hline $2 S$ & 13.2 & 9.9 & 7.8 & 7.9 \\
\hline $3 S$ & 13.4 & 10.0 & 9.3 & 8.2 \\
\hline NW & 13.3 & 9.6 & 8.2 & 8.2 \\
\hline \multicolumn{5}{|c|}{ WD-OS } \\
\hline $1 S$ & 13.2 & 6.8 & 5.9 & 5.0 \\
\hline $2 S$ & 12.0 & 7.2 & 5.5 & 4.8 \\
\hline $3 S$ & 11.6 & 7.9 & 5.0 & 4.4 \\
\hline NW & 9.1 & 6.2 & 4.2 & 4.5 \\
\hline \multicolumn{5}{|c|}{ WD-OD } \\
\hline 1S & 10.0 & 6.3 & 4.4 & 4.1 \\
\hline $2 S$ & 3.9 & 6.0 & 4.2 & 4.6 \\
\hline $3 S$ & 33.6 & 5.9 & 4.5 & 4.0 \\
\hline NW & 8.7 & 6.0 & 4.7 & 4.8 \\
\hline
\end{tabular}

time data suggest that partial letter information was an important factor in Condition WS-OS but a relatively unimportant factor in Condition WD-OD. Converging evidence can be provided by a comparison of the relevant mean fixation duration in Experiments 2 and 3. Specifically, the mean fixation duration in the NW similar conditions of Experiment 2 was $267 \mathrm{msec}$, whereas the mean fixation duration in Condition WS-OS of Experiment 3 was $307 \mathrm{msec}$ (a 40-msec difference). As discussed at the beginning of this experiment, such a difference argues strongly for the conclusion that partial letter information is being obtained in the parafovea when all of the stimuli are similar to the target. By comparison, the mean fixation duration in the NW dissimilar conditions of Experiment 2 was $223 \mathrm{msec}$, whereas the mean fixation duration in Condition WD-OD of Experiment 3 was $245 \mathrm{msec}$ (a 22-msec difference). The much smaller difference here indicates that partial letter information plays considerably less of a role when the distractors are all dissimilar to the target, a conclusion that is consistent with the previous results.

\section{GENERAL DISCUSSION}

When subjects are free to move their eyes over an array of letters, they identify relatively few letters in each fixation if near perfect accuracy is demanded of them. The actual number in the decision region varies from a low of 3-4 letters when the target is similar to the distractors to a high of 5-6 letters when the target is dissimilar to the distractors. However, it would be a mistake to conclude that only information in the decision region is relevant to the search process. In particular, the experiments reported here strongly point to the existance of a preview region that extends much farther out into the parafovea, from which additional information is obtained. The size of this preview region varies from 5-6 letters (to the right of the decision region) when the target is similar to the distractors to 7-8 letters when the target is dissimilar to the distractors. Thus, the region of effective processing varies from 9 letters when targets are embedded in similar letters to 13 letters when targets are embedded in dissimilar letters.

The general nature of the information obtained in the preview region and the impact of this information on processing in the decision region indicated that the two regions were qualitatively different from one another. Specifically, subjects were able to determine the absence of a target in the decision region but were not able to do so in the preview region even though partial letter information was clearly available in the latter region.

We should make some mention of an alternative approach we could have used to determine the number of letters that can be processed per fixation. In particular, we could have simply used tachistoscopic exposures to determine how many letters could be processed per fixation. Many studies on visual search have been conducted using brief tachistoscopic exposures that preclude the possibility of subjects' making eye movements (Duncan, 1983; Gleitman \& Jonides, 1978; Jonides \& Gleitman, 1972). Thus, one could presumably determine how many letters are processed on each fixation by examining how effectively subjects can locate a target letter as a function of the number of letters presented in the array. Experiments of this type often find that subjects are almost as fast (and accurate) in responding when 4 letters are in the

Table 14

Mean Saccade Length (in Character Spaces) in Experiment 3 as a Function of Window Size, String Size, and Background Similarity

\begin{tabular}{|c|c|c|c|c|}
\hline \multirow[b]{2}{*}{ Window Size } & \multicolumn{4}{|c|}{ String Size } \\
\hline & 2 & 4 & 6 & 8 \\
\hline \multicolumn{5}{|c|}{ WS-OS } \\
\hline $1 S$ & 2.4 & 3.0 & 3.5 & 3.3 \\
\hline $2 S$ & 2.6 & 3.2 & 3.7 & 3.4 \\
\hline $3 S$ & 2.7 & 3.1 & 3.2 & 3.2 \\
\hline NW & 3.0 & 3.3 & 3.5 & 3.5 \\
\hline \multicolumn{5}{|c|}{ WS-OD } \\
\hline 15 & 2.4 & 2.9 & 3.3 & 3.2 \\
\hline $2 S$ & 2.7 & 3.2 & 3.5 & 3.5 \\
\hline 3S & 2.9 & 3.2 & 3.4 & 3.4 \\
\hline NW & 3.0 & 3.3 & 3.5 & 3.5 \\
\hline \multicolumn{5}{|c|}{ WD-OS } \\
\hline $1 S$ & 2.9 & 4.2 & 4.4 & 4.6 \\
\hline $2 S$ & 3.0 & 4.1 & 4.8 & 5.0 \\
\hline $3 S$ & 3.5 & 4.1 & 5.4 & 5.2 \\
\hline NW & 3.9 & 4.4 & 5.4 & 5.2 \\
\hline \multicolumn{5}{|c|}{ WD-OD } \\
\hline $1 S$ & 3.7 & 4.6 & 5.5 & 5.1 \\
\hline $2 S$ & 4.2 & 4.7 & 5.1 & 5.2 \\
\hline $3 S$ & 4.2 & 4.9 & 5.5 & 5.4 \\
\hline NW & 4.3 & 5.0 & 5.4 & 5.0 \\
\hline
\end{tabular}


the display as when 2 are, but response time increases when 6 letters are present. There are two problems associated with generalizing from this type of research to the more natural situation in which subjects move their eyes. First, tachistoscopic experiments of this type can tell us nothing about the interplay between foveal and parafoveal processing. We have seen from the experiments reported here that the characteristics of the information in the parafoveal preview region affects overall search performance. Second, when stimuli are presented tachistoscopically, subjects may engage in strategies that are vastly different from those used when they are free to move their eyes. These differences in strategies could well affect conclusions that one may wish to draw concerning the number of letters that are processed per fixation.

Elsewhere, we (Rayner \& Fisher, 1987) have discussed the similarities and differences between visual search for letter targets and reading. Although the region from which information can be obtained in the two tasks is somewhat similar, the processing activities required of subjects may be quite different. Although visual search tasks may enlighten us somewhat concerning basic perceptual and oculomotor processes in both tasks (Jacobs, 1986; LevySchoen, O'Regan, Jacobs, \& Coeffe, 1984; Rayner \& Fisher, 1987), the more complex cognitive processes required in reading are not a necessary component of visual search.

In summary, we believe that the moving-window technique offers a viable alternative paradigm for studying visual search processes in situations in which subjects can move their eyes, but in which the number of letters that are available for processing can be precisely controlled. Varying the relationship between characters inside and outside the window area also appears to be very promising for investigation of critical properties of the search task. Future research using this paradigm should yield valuable insights into visual search processes.

\section{REFERENCES}

Brand, J. (1971). Classification without identification in visual search. Quarterly Journal of Experimental Psychology, 23, 178-186.

DenBuurman, R., Boersema, T., \& Gerrisen, J. F. (1981). Eye movements and the perceptual span in reading. Reading Research Quarterly, 16, 227-235.

DunCAN, J. (1983). Category effects in visual search: A failure to replicate the "Oh-Zero" phenomenon. Perception \& Psychophysics, 34, 221-232.

ENGEL, F. L. (1977). Visual conspicuity, visual search and fixation tendencies of the eye. Vision Research, 17, 95-108.

FISHER, D. L. (1984). Central capacity limits in consistent mapping, visual search tasks: Four channels or more? Cognitive Psychology, 16, 449-484.

Gleitman, H., \& Jonides, J. (1978). The effect of set on categorization in visual search. Perception \& Psychophysics, 24, 361-368.

IKEDA, M., \& SAIDA, S. (1978). Span of recognition in reading. Vision Research, 18, 83-88.

INGLING, N. (1972). Categorization: A mechanism for rapid information processing. Journal of Experimental Psychology, 94, 239-243.
JACOBS, A. M. (1986). Eye movement control in visual search: How direct is visual span control? Perception \& Psychophysics, 39, 47-58. Jonides, J., \& Gleitman, H. (1972). A conceptual categorization effect in visual search: $\mathrm{O}$ as letter or digit. Perception \& Psychophysics, $12,457-460$.

Levy-Schoen, A. (1974). Le camp d'activité du regard: Données experimentales. Année Psychologique, 74, 43-66.

Levy-Schoen, A., O'Regan, J. K., Jacobs, A. M., \& Coeffe, C. (1984). The relation between visibility span and eye movements in various scanning tasks. In A. G. Gale \& F. Johnson (Eds.), Theoretical and applied aspects of eye movement research (pp. 133-142). Amsterdam: North-Holland.

MCConkie, G. W., \& RAyner, K. (1975). The span of the effective stimulus during a fixation in reading. Perception \& Psychophysics, 17, 578-586.

NeisSER, U. (1963). Decision time without reaction time. American Journal of Psychology, 76, 376-385.

NeISSER, U. (1964). Visual search. Scientific American, 210, 94-102.

NeISSER, U., \& BELLER, H. K. (1965). Searching through word lists. British Journal of Psychology, 56, 349-358.

O'REGAN, J. K. (1979). Eye guidance in reading: Evidence for the linguistic control hypothesis. Perception \& Psychophysics, 25, 501-509.

Pollatsek, A., \& RAYNer, K. (1982). Eye movement control in reading: The role of word boundaries. Journal of Experimental Psychology: Human Perception \& Performance, 8, 817-833.

Prinz, W. (1984). Attention and sensitivity in visual search. Psychological Research, 45, 355-366.

RabbitT, P., Cumming, G., \& Vyas, S. (1978). Some errors in perceptual analysis in visual search can be detected and corrected. Quarterly Journal of Experimental Psychology, 30, 319-332.

RAYNER, K. (1984). Visual selection in reading, picture perception, and visual search: A tutorial review. In H. Bouma \& D. W. Bouwhuis (Eds.), Attention and performance $X$ (pp. 67-96). Hillsdale, NJ: Erlbaum.

RAYNER, K., \& Bertera, J. H. (1979). Reading without a fovea. Science, 206, 468-469.

RAYNER, K., FISHER, D. L. (1987). Eye movements and the perceptual span in visual search. In J. K. O'Regan \& A. Levy-Schoen (Eds.), Eye movements: From physiology to cognition (pp. 293-302). Amsterdam: Elsevier North-Holland.

RaYner, K., Inhoff, A. W., Morrison, R. E., Slowiaczex, M. L., \& Bertera, J. H. (1981). Masking of foveal and parafoveal vision during eye fixations in reading. Journal of Experimental Psychology: Human Perception \& Performance. 7, 167-179.

Rayner, K., Well, A. D., \& Pollatsek, A. (1980). Asymmetry of the effective visual field in reading. Perception \& Psychophysics, 27, 537-544.

Rayner, K., Well, A. D., Pollatsek, A., \& Bertera, J. H. (1982). The availability of useful information to the right of fixation in reading. Perception \& Psychophysics, 31, 537-550.

SCHNEIDER, W., SHIFFrin, R. M. (1977). Controlled and automatic human information processing: 1 . Detection, search, and attention. Psychological Review, 84, 1-66.

Underwoon, N. R., \& McConkIE, G. W. (1985). Perceptual span for letter distinctions during reading. Reading Research Quarterly, 20, 153-162.

\section{NOTES}

1. The speed with which subjects in our experiments searched for the target letter was quite similar to that obtained in other studies (Brand, 1971; Ingling, 1972; Neisser, 1963; Rabbit, Cumming, \& Vyas, 1978), so the fact that our subjects searched horizontally while others have searched vertically is probably irrelevant. When the search times in these other studies was converted to milliseconds per character, the rates ranged between 40 and 100 msec per character (which is about the same as our range), depending upon the exact nature of the condition.

2. Experiments in which subjects searched vertically through an array have likewise demonstrated that the area of processing is asymmet- 
ric, with more information obtained from below the fixation than from above (Prinz, 1984).

3. Because, in some cases, the estimate of the minimum size of the region of effective processing varied with the string size, we used, as our estimate of the size of this region, the maximum of the minimums. For example, if the smallest estimate of the minimum size of the region of effective processing occurred with strings of size 2 and the largest estimate of the minimum size of the region occurred with string size 8, we report the latter estimate. This is because we are interested in a conservative estimate of the size of the region (and so we chose a minimum measure) under circumstances most favorable to its existence (and so we chose the maximum of the minimums). In any case, the range of estimates of the minimum size of the region of effective processing was very small.

4. Although the data reported here focus on saccade length from targetabsent lines, it should be noted that examination of the length of the saccade to the target (or close to the target) in cases when a target was present in the display revealed that, across the three experiments, its average length did not differ appreciably from that of the earlier saccades on that trial or from the mean saccade length when a target was not present. This reinforces the argument that saccade length reflects the number of letters in the decision region and that the subject moves to a position outside the decision region on each saccade.

5. It is important to note that when only trials in which no right-toleft saccades occurred were examined, the results were the same as those reported in the text in all three experiments. We have included trials with right-to-left saccades since they do contribute to the overall search time that would be obtained if eye movements were not examined.

(Manuscript received March 17, 1986; revision accepted for publication February 6, 1987.) 